\title{
Improved conditions for neutral delay systems with novel inequalities
}

\author{
Lianglin Xionga,*, Jun Cheng ${ }^{\mathrm{b}}$, Xinzhi Liuc ${ }^{\mathrm{c}}$, Tao Wü \\ a School of Mathematics and Computer Science, Yunnan Minzu University, Kunming, 650500, China. \\ ${ }^{b}$ School of Science, Hubei University for Nationalities, Enshi, 445000, China. \\ ${ }^{c}$ Department of Applied Mathematics, University of Waterloo, Waterloo, Ontario, Canada N2L 3G1. \\ Communicated by R. Saadati
}

\begin{abstract}
This paper studies the stability problem of a class of neutral delay systems. It firstly establishes two novel integral inequalities, which are better than the same type inequalities found in the literature. Then it derives, by using the new inequalities and the Lyapunov functional method, some sufficient delay-dependent conditions for asymptotic stability of the neutral delay systems. Three numerical examples are provided to illustrate the advantage and effectiveness of the obtained results. (C)2017 All rights reserved.
\end{abstract}

Keywords: New integral inequality, neutral delay system, delay-dependent stability, Lyapunov functional. 2010 MSC: 34D05, 26D10, 34k06.

\section{Introduction}

In the past decades, a great deal of attention have been drawn to the delay-dependent stability criteria of neutral delay systems (see, e.g., $[1-4,7-9,11,12,15,17,18])$. Various interesting methods have been introduced to obtain delay-dependent stability conditions for neutral delay systems, such as model transformation approach [8], delay partitioning technique [2], discretized Lyapunov functional method [11], free-weighting matrix approach [9, 12], and integral inequality method [1]. With the help of these approaches, improved delay-dependent stability conditions have been presented gradually, such as $[3,11,15]$ and the references therein. It is known that Jensen's like inequalities have played an important role in obtaining delay-dependent stability conditions.

However, Jensen's like inequality inevitably introduces some undesirable conservatism. It has been improved by [13] which dealt with single integral and double integral terms. As a result, a new class of inequalities are presented, which produce tighter bounds than what the Jensen inequality produces in [13]. Very recently, a less conservative inequality was introduced based on the free-matrix ideal [21, 22]. They provided a better condition on the single integral inequality than [13]. On the other hand, research on

\footnotetext{
${ }^{*}$ Corresponding author

Email addresses: lianglin_5418@126.com (Lianglin Xiong), jcheng6819@126.com (Jun Cheng), xinzhi.liu@uwaterloo.ca (Xinzhi Liu), 1175908375@qq. com (Tao Wu)
}

doi:10.22436/jnsa.010.05.03 
double integral inequality and triple integral inequality should not be ignored, because such inequalities may play an important role in obtaining delay-dependent stability condition for neutral delay systems.

Motivated by the above discussion, we investigate, in this paper, the conventional neutral systems to obtain delay-dependent stability by using the new introduced inequalities. The rest of the paper is organized as follows. In Section 2, a new class of inequalities are provided. In Section 3, stability analysis for neutral delay systems is presented. Sufficient conditions are formed in terms of LMIs, which can be easily calculated by Matlab control toolbox. Many cases are compared in the tables based on the conventional numerical examples, which are used to show the validity and less conservativeness of our approach in Section 4. A conclusion is given in Section 5.

\section{Preliminaries}

Let $R^{n}$ denote the $n$-dimensional Euclidean space and $R^{m \times n}$ is the set of all $m \times n$ real matrices. For a real symmetric matrix $X, X>0(X \geqslant 0)$ means that $X$ is positive definite (semi-positive definite). $I$ is the identity matrix of appropriate dimensions. The superscript " $T$ " stands for the transpose of a matrix or a vector. I is the identity matrix of appropriate dimensions. We shall first establish two novel inequalities for late use.

Lemma 2.1. For a given symmetric positive definite matrix $\mathrm{R}>0$ and any differentiable function $\mathrm{x}:[\mathrm{a}, \mathrm{b}] \rightarrow \mathbb{R}^{\mathrm{n}}$, the following inequality holds:

$$
\int_{a}^{b} \int_{\theta}^{b} \int_{u}^{b} \dot{x}^{\top}(s) R \dot{x}(s) d s d u d \theta \geqslant \frac{6}{(b-a)^{3}} A^{\top} R A+\frac{5(b-a)}{2} B^{\top} R B
$$

where

$$
\begin{aligned}
& A=\frac{(b-a)^{2}}{2} x(b)-\int_{a}^{b} \int_{\theta}^{b} x(u) d u d \theta \\
& B=x(b)+\frac{6}{(b-a)^{2}} \int_{a}^{b} \int_{\theta}^{b} x(u) d u d \theta-\frac{16}{(b-a)^{3}} \int_{a}^{b} \int_{\theta}^{b} \int_{u}^{b} x(s) d s d u d \theta .
\end{aligned}
$$

Proof. For a continuously differentiable function $x(t), t \in[a, b]$, define the function $z$ by

$$
z(s)=\dot{x}(s)-\frac{6}{(b-a)^{3}} A-\frac{5 g(s)}{(b-a)^{2}} B,
$$

where

$$
g(s)=4 s-3 b-a,
$$

$A$ and $B$ are defined in Lemma 2.1. It is easy to obtain that

$$
\begin{aligned}
& \int_{a}^{b} \int_{\theta}^{b} \int_{u}^{b} \dot{x}(s) d s d u d \theta=\frac{(b-a)^{2}}{2} x(b)-\int_{a}^{b} \int_{\theta}^{b} x(u) d u d \theta=A, \\
& \int_{a}^{b} \int_{\theta}^{b} \int_{u}^{b} g(s) d s d u d \theta=0, \\
& \int_{a}^{b} \int_{\theta}^{b} \int_{u}^{b} g^{2}(s) d s d u d \theta=\frac{(b-a)^{5}}{10}, \quad \int_{a}^{b} \int_{\theta}^{b} \int_{u}^{b} g(s) \dot{x}(s) d s d u d \theta=\frac{(b-a)^{3}}{2} B .
\end{aligned}
$$

With $R>0$, the computation of $\int_{a}^{b} \int_{\theta}^{b} \int_{u}^{b} z^{\top}(s) R z(s) d s d u d \theta$ leads to

$$
\int_{a}^{b} \int_{\theta}^{b} \int_{u}^{b} z^{\top}(s) R z(s) d s d u d \theta=\int_{a}^{b} \int_{\theta}^{b} \int_{u}^{b} \dot{x}^{\top}(s) R \dot{x}(s) d s d u d \theta-\frac{6}{(b-a)^{3}} A^{\top} R A-\frac{5(b-a)}{2} B^{\top} R B>0 .
$$

Clearly, the above inequality is equivalent to inequality (2.1). This completes the proof. 
Remark 2.2. Compared to the conventional Jensen's like inequality for triple integral, our inequality has additional positive term $\frac{5 h}{2} B^{T} R B$. Consequently, this inequality is less conservative than the inequality given in [1].

Lemma 2.3. For a given symmetric positive definite matrix $\mathrm{R}>0$ and any differentiable function $\mathrm{x}:[\mathrm{a}, \mathrm{b}] \rightarrow \mathrm{R}^{\mathrm{n}}$, and $\mathrm{N}_{1}, \mathrm{~N}_{2}, \mathrm{~N}_{3} \in \mathbb{R}^{4 \mathrm{n} \times \mathrm{n}}$, then the following inequality holds:

$$
-\int_{a}^{b} \int_{\theta}^{b} \dot{x}^{\top}(s) R \dot{x}(s) d s d \theta \leqslant v^{\top} \Omega v
$$

where

$$
\begin{aligned}
& \Omega=\frac{(b-a)^{2}}{2} N_{1} R^{-1} N_{1}^{\top}+\frac{(b-a)^{2}}{4} N_{2} R^{-1} N_{2}^{\top}+\frac{(b-a)^{2}}{6} N_{3} R^{-1} N_{3}^{\top}+(b-a) \operatorname{sym}\left\{N_{1} \Pi_{1}+N_{2} \Pi_{2}+N_{3} \Pi_{3}\right\}, \\
& \Pi_{1}=e_{1}-e_{2}, \quad \Pi_{2}=e_{1}+2 e_{2}-6 e_{3}, \quad \Pi_{3}=e_{1}-3 e_{2}+24 e_{3}-60 e_{4}, \\
& v=\left[\begin{array}{lll}
x^{\top}(b) & \frac{1}{b-a} \int_{a}^{b} x^{\top}(s) d s \quad \frac{1}{(b-a)^{2}} \int_{a}^{b} \int_{\theta}^{b} x^{\top}(s) d s d \theta \frac{1}{(b-a)^{3}} \int_{a}^{b} \int_{\theta}^{b} \int_{u}^{b} x^{\top}(s) d s d u d \theta
\end{array}\right]^{\top}, \\
& e_{i}=[0 \cdots \underbrace{I}_{i} \cdots 0] \in \mathbb{R}^{m n \times n}(i=1, \cdots, 4) .
\end{aligned}
$$

Proof. Define

$$
\begin{aligned}
N & =\left[\begin{array}{lll}
N_{1}^{\top} & N_{2}^{\top} & N_{3}^{\top}
\end{array}\right]^{\top}, & \zeta(s) & =\left[\begin{array}{lll}
v^{\top} & f_{1}(s) v^{\top} & f_{2}(s) v^{\top}
\end{array}\right]^{\top}, \\
f_{1}(s) & =\frac{3 s-2 b-a}{b-a}, & f_{2}(s) & =\frac{10 s^{2}-4(3 b+2 a) s+3 b^{2}+a^{2}+6 a b}{(b-a)^{2}} .
\end{aligned}
$$

It is easy to see that

$$
-2 \zeta^{\top}(s) N \dot{x}(s)=\zeta^{\top}(s) N R^{-1} N^{\top} \zeta(s)+\dot{x}^{\top}(s) R \dot{x}(s) .
$$

Integrating (2.3) from $a$ to $b$ yields

$$
\begin{aligned}
& -2(b-a) v^{\top} N_{1}\left(e_{1}-e_{2}\right) v-2(b-a) v^{\top} N_{2}\left(e_{1}+2 e_{2}-6 e_{3}\right) v-2(b-a) v^{\top} \\
& \times N_{3}\left(e_{1}-3 e_{2}+24 e_{3}-60 e_{4}\right) v \\
& \quad \leqslant \frac{(b-a)^{2}}{2} N_{1} R^{-1} N_{1}^{\top}+\frac{(b-a)^{2}}{4} N_{2} R^{-1} N_{2}^{\top}+\frac{(b-a)^{2}}{6} N_{3} R^{-1} N_{3}^{\top}+\int_{a}^{b} \int_{\theta}^{b} \dot{x}^{\top}(s) R \dot{x}(s) d s d \theta .
\end{aligned}
$$

Rearranging (2.2) yields (2.4), and this completes the proof.

Remark 2.4. Two functions $f_{1}(s)$ and $f_{2}(s)$ play very important role in the proof of Lemma 2.3. Less conservative results are obtained by employing the free matrices $N_{1}, N_{2}$ and $N_{3}$ to deal with the relationships among $x(b), \int_{a}^{b} x(s) d s, \int_{a}^{b} \int_{\theta}^{b} x(s) d s d \theta$ and $\int_{a}^{b} \int_{\theta}^{b} \int_{u}^{b} x(s) d s d u d \theta$. In fact, the inequality (25) in Lemma 5.1 in [7] is a special case of Lemma 2.3 by setting $N_{1}=-\frac{2}{b-a} \Pi_{1}^{\top} R, N_{2}=-\frac{4}{b-a} \Pi_{2}^{T} R$, and $N_{3}=0$. Moreover, the inequality (4) of Lemma 2.3 in [23] is also a special case of Lemma 2.3 by setting $N_{1}=-\frac{2}{b-a} \Pi_{1}^{T} R$, $N_{2}=-\frac{4}{b-a} \Pi_{2}^{\top} R$, and $N_{3}=-\frac{6}{b-a} \Pi_{3}^{\top} R$. Different from the inequality of Lemma 1 in [22], this inequality is a double integral inequality, which could be used to reduce the conservativeness of the delay-dependent stability conditions.

\section{Main results}

Consider the following neutral delay system

$$
\left\{\begin{array}{l}
\dot{x}(t)-C \dot{x}(t-\tau)=A x(t)+B x(t-h), \\
x\left(t_{0}+\theta\right)=\varphi(\theta), \forall \theta \in[-\rho, 0]
\end{array}\right.
$$


where $x(t) \in \mathbb{R}^{n}$ is the state vector, $\tau$ and $h$ are time-delays, $\rho=\max \{\tau, h\}, \varphi(\theta)$ is the initial condition function, and $A, B, C$ are known matrices. In this paper, we always assume that the spectral radius of $C$ less than 1.

System (3.1) has been well studied in the literature and many results are available. However, by using the novel inequalities developed earlier, we can obtain much less conservative conditions than the existing results.

Theorem 3.1. System (3.1) is asymptotically stable if there exist positive definite matrices $W_{1}, W_{2}, Q_{1}, Q_{2}, R_{1}, R_{2}$, symmetric matrices $X \in \mathbb{R}^{8 n \times 8 n}, \mathfrak{R} \in \mathbb{R}^{2 n \times 2 n}$, and any matrices $M_{i}, N_{i} \in \mathbb{R}^{4 n \times n}(i \in 1,2,3)$ with appropriate dimensions satisfying the following inequalities

$$
\begin{aligned}
& \Psi=\widetilde{\mathrm{G}}_{1}^{\top} X \widetilde{\mathrm{G}}_{1}+\tau e_{3}^{\top} \mathrm{Q}_{1} e_{3}+3 \tau\left(e_{3}-2 e_{5}\right)^{\top} \mathrm{Q}_{1}\left(e_{3}-2 e_{5}\right)+5 \tau\left(e_{3}-6 e_{5}+12 e_{7}\right)^{\top} \mathrm{Q}_{1}\left(e_{3}-6 e_{5}+12 e_{7}\right) \\
& +h e_{4}^{\top} Q_{2} e_{4}+3 h\left(e_{4}-2 e_{6}\right)^{\top} Q_{2}\left(e_{4}-2 e_{6}\right)+5 h\left(e_{4}-6 e_{6}+12 e_{8}\right)^{\top} Q_{1}\left(e_{4}-6 e_{6}+12 e_{8}\right)
\end{aligned}
$$

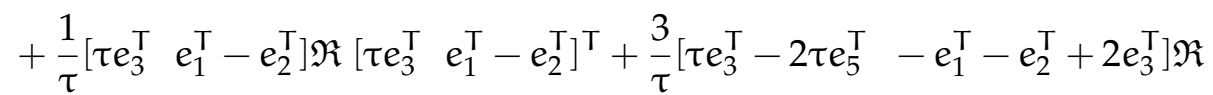

$$
\begin{aligned}
& \times\left[\tau e_{3}^{T}-2 \tau e_{5}^{T}-e_{1}^{T}-e_{2}^{T}+2 e_{3}^{T}\right]^{T}+\frac{5}{\tau}\left[\tau e_{3}^{T}-6 \tau e_{5}^{T}+12 \tau e_{7}^{T} \quad e_{1}^{T}-e_{2}^{T}-6 e_{3}^{T}+12 \tau e_{5}^{T}\right] \Re \\
& \times\left[\tau e_{3}^{\top}-6 \tau e_{5}^{T}+12 \tau e_{7}^{\top} e_{1}^{\top}-e_{2}^{T}-6 e_{3}^{T}+12 \tau e_{5}^{T}\right]^{\top}+2 \tau^{3} e_{5}^{\top} W_{1} e_{5}+16 \tau^{3}\left(e_{5}-3 e_{7}\right)^{\top} W_{1}\left(e_{5}-3 e_{7}\right) \\
& +6 \tau\left(\frac{1}{2} e_{1}-e_{5}\right)^{\top} R_{1}\left(\frac{1}{2} e_{1}-e_{5}\right)+2 h^{3} e_{6}^{\top} W_{2} e_{6}+16 h^{3}\left(e_{6}-3 e_{8}\right)^{\top} W_{2}\left(e_{6}-3 e_{8}\right) \\
& +6 h\left(\frac{1}{2} e_{1}-e_{6}\right)^{\mathrm{T}} R_{2}\left(\frac{1}{2} e_{1}-e_{6}\right)+\frac{5 \tau}{2}\left(e_{1}+6 e_{5}-16 e_{7}\right)^{\mathrm{T}} R_{1}\left(e_{1}+6 e_{5}-16 e_{7}\right) \\
& +\frac{5 h}{2}\left(e_{1}+6 e_{6}-16 e_{8}\right)^{\top} R_{2}\left(e_{1}+6 e_{6}-16 e_{8}\right)>0, \\
& e_{i}=[0 \cdots \underbrace{I}_{i} \cdots 0] \in \mathbb{R}^{8 n \times n}(i=1, \cdots, 8) \text {, } \\
& \varphi=\bar{G}_{1}^{\top} X \widetilde{G}_{1}+\widetilde{G}_{1}^{\top} X \bar{G}_{1}+\tau^{2} e_{1}^{\top} W_{1} e_{1}+h^{2} e_{1}^{\top} W_{2} e_{1}+\frac{\tau^{2}}{2} e_{0}^{\top} R_{1} e_{0}+\frac{h^{2}}{2} e_{0}^{\top} R_{2} e_{0}+e_{1}^{\top} Q_{1} e_{1}-e_{2}^{\top} Q_{1} e_{2} \\
& +\left(\begin{array}{l}
e_{1} \\
e_{0}
\end{array}\right)^{\top} R\left(\begin{array}{l}
e_{1} \\
e_{0}
\end{array}\right)-\left(\begin{array}{l}
e_{2} \\
e_{4}
\end{array}\right)^{\top} R\left(\begin{array}{l}
e_{2} \\
e_{4}
\end{array}\right)-\tau^{2} e_{5}^{\top} W_{1} e_{5}-3 \tau^{2}\left(e_{5}-2 e_{7}\right)^{\top} W_{1}\left(e_{5}-2 e_{7}\right) \\
& -5 \tau^{2}\left(e_{5}-6 e_{7}+12 e_{9}\right)^{\top} W_{1}\left(e_{5}-6 e_{7}+12 e_{9}\right)-h^{2} e_{6}^{\top} W_{2} e_{6}-3 h^{2}\left(e_{6}-2 e_{8}\right)^{\top} W_{2}\left(e_{6}-2 e_{8}\right) \\
& -5 h^{2}\left(e_{6}-6 e_{8}+12 e_{10}\right)^{\mathrm{T}} W_{1}\left(e_{6}-6 e_{8}+12 e_{10}\right)+\Omega_{1}^{\top} K_{1} \Omega_{1}+\Omega_{2}^{\top} K_{2} \Omega_{2}<0, \\
& e_{i}=[0 \cdots \underbrace{I}_{i} \cdots 0] \in \mathbb{R}^{10 n \times n}(i=1, \cdots, 10) \text {, }
\end{aligned}
$$

where

$$
\begin{aligned}
& e_{0}=A e_{1}+B e_{3}+C e_{4}, \quad \Omega_{1}=\left(e_{1}, e_{5}, e_{7}, e_{9}\right)^{\top}, \Omega_{2}=\left(e_{1}, e_{6}, e_{8}, e_{10}\right)^{\top}, \Pi_{1}=e_{1}-e_{5}, \Pi_{2}=e_{1}+2 e_{5}-6 e_{7}, \\
& \Pi_{3}=e_{1}-3 e_{5}+24 e_{7}-60 e_{9}, \Pi_{4}=e_{1}-e_{6}, \Pi_{5}=e_{1}+2 e_{6}-6 e_{8}, \Pi_{6}=e_{1}-3 e_{6}+24 e_{8}-60 e_{10},
\end{aligned}
$$

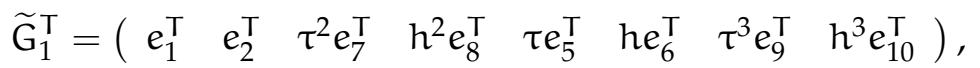

$$
\begin{aligned}
& \bar{G}_{1}=\left[e_{0}^{\top} e_{4}^{\top} \tau\left(e_{1}-e_{5}\right)^{\top} h\left(e_{1}-e_{6}\right)^{\top}\left(e_{1}-e_{2}\right)^{\top}\left(e_{1}-e_{3}\right)^{\top} \tau^{2}\left(\frac{e_{1}}{2}-e_{7}\right)^{\top} h^{2}\left(\frac{e_{1}}{2}-e_{8}\right)^{\top}\right], \\
& \mathrm{K}_{1}=\frac{\tau^{2}}{2} \mathrm{~N}_{1} R_{1}^{-1} \mathrm{~N}_{1}^{\top}+\frac{\tau^{2}}{4} \mathrm{~N}_{2} R_{1}^{-1} \mathrm{~N}_{2}^{\top}+\frac{\tau^{2}}{6} \mathrm{~N}_{3} R_{1}^{-1} N_{3}^{\top}+\tau \cdot \operatorname{sym}\left\{N_{1} \Pi_{1}+N_{2} \Pi_{2}+N_{3} \Pi_{3}\right\}, \\
& K_{2}=\frac{h^{2}}{2} M_{1} R_{2}^{-1} M_{1}^{\top}+\frac{h^{2}}{4} M_{2} R_{2}^{-1} M_{2}^{\top}+\frac{h^{2}}{6} M_{3} R_{2}^{-1} M_{3}^{\top}+h \cdot \operatorname{sym}\left\{M_{1} \Pi_{4}+M_{2} \Pi_{5}+M_{3} \Pi_{6}\right\} .
\end{aligned}
$$


Proof. Construct a Lyapunov functional candidate as

$$
\begin{aligned}
V\left(x_{t}, t\right)= & G_{1}^{T} X G_{1}+\int_{t-\tau}^{t} x^{\top}(s) Q_{1} x(s) d s+\int_{t-h}^{t} x^{\top}(s) Q_{2} x(s) d s+\int_{t-\tau}^{t}\left(\begin{array}{c}
x(s) \\
\dot{x}(s)
\end{array}\right)^{\top} \mathfrak{R}\left(\begin{array}{c}
x(s) \\
\dot{x}(s)
\end{array}\right) d s \\
& +\int_{t-\tau}^{t} \int_{\beta}^{t} x^{\top}(\alpha)\left(\tau W_{1}\right) x(\alpha) d \alpha d \beta+\int_{t-h}^{t} \int_{\beta}^{t} x^{\top}(\alpha)\left(h W_{2}\right) x(\alpha) d \alpha d \beta \\
& +\int_{t-\tau}^{t} \int_{\theta}^{t} \int_{\lambda}^{t} \dot{x}^{\top}(s) R_{1} \dot{x}(s) d s d \lambda d \theta+\int_{t-h}^{t} \int_{\theta}^{t} \int_{\lambda}^{t} \dot{x}^{\top}(s) R_{2} \dot{x}(s) d s d \lambda d \theta
\end{aligned}
$$

where $X, Q_{1}, Q_{2}, W_{1}, W_{2}, R_{1}, R_{2}, \Re$ are defined in Theorem 3.1, and

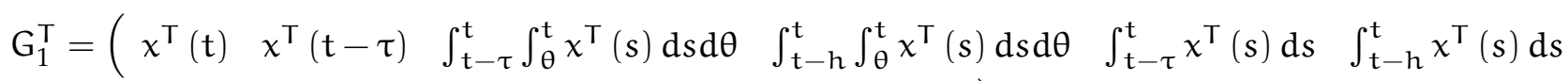

$$
\begin{aligned}
& \left.\int_{t-\tau}^{t} \int_{\theta}^{t} \int_{u}^{t} x^{\top}(s) d s d u d \theta \quad \int_{t-h}^{t} \int_{\theta}^{t} \int_{u}^{t} x^{\top}(s) d s d u d \theta\right)=\xi^{\top} \widetilde{G}_{1}^{\top}
\end{aligned}
$$

with

$$
\xi^{\top}=\left(\begin{array}{l}
x^{\top}(t), x^{\top}(t-\tau), x^{\top}(t-h), \dot{x}^{\top}(t-\tau), \frac{1}{\tau} \int_{t-\tau}^{t} x^{\top}(s) d s, \frac{1}{h} \int_{t-h}^{t} x^{\top}(s) d s, \frac{1}{\tau^{2}} \int_{t-\tau}^{t} \int_{\theta}^{t} x^{\top}(s) d s d \theta, \\
\frac{1}{h^{2}} \int_{t-h}^{t} \int_{\theta}^{t} x^{\top}(s) d s d \theta, \frac{1}{\tau^{3}} \int_{t-\tau}^{t} \int_{\theta}^{t} \int_{u}^{t} x^{\top}(s) d s d u d \theta, \frac{1}{h^{3}} \int_{t-h}^{t} \int_{\theta}^{t} \int_{u}^{t} x^{\top}(s) d s d u d \theta
\end{array}\right) .
$$

First, with Lemma 2.1, Lemma 2.3, and some inequalities in [7], the Lyapunov functionals $V$ can be scaled as

$$
\begin{aligned}
& \mathrm{V} \geqslant \mathrm{G}_{1}^{\top} X \mathrm{G}_{1}+\xi^{\top}\left\{\tau e_{5}^{\top} \mathrm{Q}_{1} e_{5}+3 \tau\left(e_{5}-2 e_{7}\right)^{\top} \mathrm{Q}_{1}\left(e_{5}-2 e_{7}\right)+5 \tau\left(e_{5}-6 e_{7}+12 e_{9}\right)^{\top} \mathrm{Q}_{1}\left(e_{5}-6 e_{7}+12 e_{9}\right)\right. \\
& +h e_{6}^{\top} \mathrm{Q}_{2} e_{6}+3 \mathrm{~h}\left(e_{6}-2 e_{8}\right)^{\top} \mathrm{Q}_{2}\left(e_{6}-2 e_{8}\right)+5 \mathrm{~h}\left(e_{6}-6 e_{8}+12 e_{10}\right)^{\top} \mathrm{Q}_{1}\left(e_{6}-6 e_{8}+12 e_{10}\right)
\end{aligned}
$$

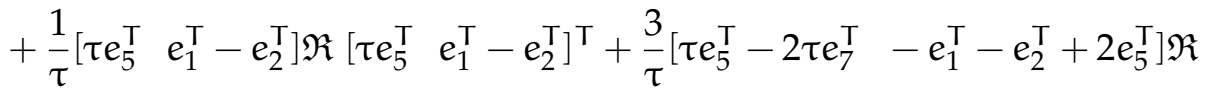

$$
\begin{aligned}
& \times\left[\tau e_{5}^{T}-2 \tau e_{7}^{T}-e_{1}^{T}-e_{2}^{T}+2 e_{5}^{T}\right]^{T}+\frac{5}{\tau}\left[\tau e_{5}^{T}-6 \tau e_{7}^{T}+12 \tau e_{9}^{T} \quad e_{1}^{\top}-e_{2}^{T}-6 e_{5}^{T}+12 \tau e_{7}^{T}\right] \Re \\
& \times\left[\tau e_{5}^{T}-6 \tau e_{7}^{T}+12 \tau e_{9}^{T} \quad e_{1}^{\top}-e_{2}^{T}-6 e_{5}^{T}+12 \tau e_{7}^{T}\right]^{T}+2 \tau^{3} e_{7}^{\top} W_{1} e_{7}+16 \tau^{3}\left(e_{7}-3 e_{9}\right)^{T} W_{1}\left(e_{7}-3 e_{9}\right) \\
& \left.+2 h^{3} e_{8}^{\top} W_{2} e_{8}+16 h^{3}\left(e_{8}-3 e_{10}\right)^{\top} W_{2}\left(e_{8}-3 e_{10}\right)\right\} \times \xi+\xi^{\top}\left\{6 \tau\left(\frac{1}{2} e_{1}-e_{7}\right)^{\top} R_{1}\left(\frac{1}{2} e_{1}-e_{7}\right)\right. \\
& \left.+6 h\left(\frac{1}{2} e_{1}-e_{8}\right)^{\mathrm{T}} R_{2}\left(\frac{1}{2} e_{1}-e_{8}\right)\right\} \xi+\xi^{\top}\left\{\frac{5 \tau}{2}\left(e_{1}-6 e_{7}+16 e_{9}\right)^{\top} R_{1}\left(e_{1}-6 e_{7}+16 e_{9}\right)\right. \\
& \left.+\frac{5 h}{2}\left(e_{1}-6 e_{8}+16 e_{10}\right)^{\mathrm{T}} R_{2}\left(e_{1}-6 e_{8}+16 e_{10}\right)\right\} \xi .
\end{aligned}
$$

According to the inequality (3.2), the constructed functional (3.3) is positive definite. Then, with Lemma 2.1, Lemma 2.1 and some inequalities in [7] the time derivative of $V(t)$ along the trajectories of equation (3.1) can be computed as follows:

$$
\begin{aligned}
\dot{V}\left(x_{t}, t\right) \leqslant & \xi^{\top}\left[\bar{G}_{1}^{\top} X G_{1}+G_{1}^{\top} X \bar{G}_{1}+e_{0}^{\top}\left(\tau^{2}\left(W_{1}+\frac{R_{1}}{2}\right)+h^{2}\left(W_{2}+\frac{R_{2}}{2}\right)\right) e_{0}\right. \\
& \left.+e_{1}^{\top}\left(Q_{1}+Q_{2}\right) e_{1}-e_{2}^{\top} Q_{1} e_{2}-e_{3}^{\top} Q_{2} e_{3}\right] \xi+\xi^{\top}\left[\left(\begin{array}{c}
e_{1} \\
e_{0}
\end{array}\right)^{\top} \mathfrak{R}\left(\begin{array}{c}
e_{1} \\
e_{0}
\end{array}\right)-\left(\begin{array}{c}
e_{2} \\
e_{4}
\end{array}\right)^{\top} \mathfrak{R}\left(\begin{array}{c}
e_{2} \\
e_{4}
\end{array}\right)\right] \xi \\
& -\xi^{\top}\left[\tau^{2} e_{5}^{\top} W_{1} e_{5}+3 \tau^{2}\left(e_{5}-2 e_{7}\right)^{\top} W_{1}\left(e_{5}-2 e_{7}\right)\right] \xi \\
& -\xi^{\top}\left[5 \tau^{2}\left(e_{5}-6 e_{7}+12 e_{9}\right)^{\top} W_{1}\left(e_{5}-6 e_{7}+12 e_{9}\right)\right] \xi \\
& -\xi^{\top}\left[h^{2} e_{6}^{\top} W_{2} e_{6}+3 h^{2}\left(e_{6}-2 e_{8}\right)^{\top} W_{2}\left(e_{6}-2 e_{8}\right)\right] \xi \\
& -\xi^{\top}\left[5 h^{2}\left(e_{6}-6 e_{8}+12 e_{10}\right)^{\top} W_{1}\left(e_{6}-6 e_{8}+12 e_{10}\right)-\Omega_{1}^{\top} K_{1} \Omega_{1}-\Omega_{2}^{\top} K_{2} \Omega_{2}\right] \xi=\xi^{\top} \varphi \xi .
\end{aligned}
$$

Based on the conditions of Theorem 3.1, we can obtain that $\dot{V}<0$. Accordingly, system (3.1) is asymptotically stable, which concludes the proof. 
Remark 3.2. In the process of the above proof, $x(t-h)$ and $\dot{x}(t-\tau)$ do not appear as the $V$ is considered. Therefore, the number of vector components in (3.2) of Theorem 3.1 is less than that of (3.4). When $\tau=h$, following the same line of the proof for Theorem 3.1, one can easily obtain another delay-dependent condition. In this case, it is better to use another new condition to compute the bounds of delay for the neutral systems, because many unnecessary matrix variables would be involved if we use the result in Theorem 3.1.

Theorem 3.3. System (3.1) with $\tau=\mathrm{h}$ is asymptotically stable if there exist positive definite matrices $\mathrm{W}_{1}, \mathrm{Q}_{1}$, $\mathrm{R}_{1}$, symmetric matrices $X \in \mathbb{R}^{5 n \times 5 n}, \mathfrak{R} \in \mathbb{R}^{2 \mathrm{n} \times 2 \mathrm{n}}$, and any matrices $\widetilde{M}_{i} \in \mathbb{R}^{4 \mathrm{n} \times \mathrm{n}}(\mathfrak{i} \in 1,2,3)$ with appropriate dimensions satisfying the following inequalities

$$
\begin{aligned}
& \Psi=\widetilde{\mathrm{G}}_{2}^{\top} X \widetilde{\mathrm{G}}_{2}+\tau e_{3}^{\top} \mathrm{Q}_{1} e_{3}+3 \tau\left(e_{3}-2 e_{4}\right)^{\top} \mathrm{Q}_{1}\left(e_{3}-2 e_{4}\right)+5 \tau\left(e_{3}-6 e_{4}+12 e_{5}\right)^{\top} \mathrm{Q}_{1}\left(e_{3}-6 e_{4}+12 e_{5}\right)
\end{aligned}
$$

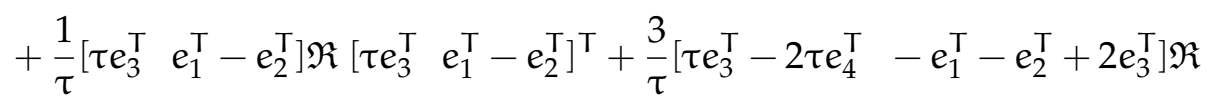

$$
\begin{aligned}
& \times\left[\tau e_{3}^{T}-2 \tau e_{4}^{T}-e_{1}^{T}-e_{2}^{\top}+2 e_{3}^{T}\right]^{T}+\frac{5}{\tau}\left[\tau e_{3}^{T}-6 \tau e_{4}^{T}+12 \tau e_{5}^{\top} \quad e_{1}^{\top}-e_{2}^{T}-6 e_{3}^{T}+12 \tau e_{4}^{\top}\right] \Re \\
& \times\left[\tau e_{3}^{T}-6 \tau e_{4}^{T}+12 \tau e_{5}^{T} \quad e_{1}^{\top}-e_{2}^{T}-6 e_{3}^{T}+12 \tau e_{4}^{T}\right]^{T}+2 \tau^{3} e_{4}^{T} W_{1} e_{4}+16 \tau^{3}\left(e_{4}-3 e_{5}\right)^{T} W_{1}\left(e_{4}-3 e_{5}\right) \\
& +6 \tau\left(\frac{1}{2} e_{1}-e_{4}\right)^{\mathrm{T}} R_{1}\left(\frac{1}{2} e_{1}-e_{4}\right)+\frac{5 \tau}{2}\left(e_{1}+6 e_{4}-16 e_{5}\right)^{\mathrm{T}} R_{1}\left(e_{1}+6 e_{4}-16 e_{5}\right)>0, \\
& e_{i}=\left[\begin{array}{lll}
0 & \cdots & \underbrace{I}_{i}
\end{array} \cdots 0\right] \in \mathbb{R}^{5 n \times n}(i=1, \cdots, 5) \text {, } \\
& \varphi=\overline{\mathrm{G}}_{3}^{\top} X \widetilde{\mathrm{G}}_{3}+\widetilde{\mathrm{G}}_{3}^{\top} X \overline{\mathrm{G}}_{3}+e_{1}^{\top} \mathrm{Q}_{1} e_{1}-e_{2}^{\top} \mathrm{Q}_{1} e_{2}+\tau^{2} e_{1}^{\top} W_{1} e_{1}-\tau^{2} e_{4}^{\top} W_{1} e_{4}+\frac{\tau^{2}}{2} e_{0}^{\top} R_{1} e_{0} \\
& +\left(\begin{array}{l}
e_{1} \\
e_{0}
\end{array}\right)^{\top} R\left(\begin{array}{l}
e_{1} \\
e_{0}
\end{array}\right)-\left(\begin{array}{l}
e_{2} \\
e_{3}
\end{array}\right)^{\top} R\left(\begin{array}{l}
e_{2} \\
e_{3}
\end{array}\right)-\tau^{2} e_{4}^{\top} W_{1} e_{4}-3 \tau^{2}\left(e_{4}-2 e_{5}\right)^{\top} W_{1}\left(e_{4}-2 e_{5}\right) \\
& -5 \tau^{2}\left(e_{4}-6 e_{5}+12 e_{6}\right)^{\mathrm{T}} W_{1}\left(e_{4}-6 e_{5}+12 e_{6}\right)+\Omega_{3}^{\top} \widetilde{K}_{1} \Omega_{3}<0, \\
& e_{i}=[0 \cdots \underbrace{I}_{i} \cdots 0] \in \mathbb{R}^{6 n \times n}(i=1, \cdots, 6) \text {, }
\end{aligned}
$$

where

$$
\begin{aligned}
& \widetilde{\mathrm{G}}_{2}^{\top}=\left(\begin{array}{llllll}
e_{1}^{\top} & e_{2}^{\top} & e_{3}^{\top} & \tau e_{4}^{\top} & \tau^{2} e_{5}^{\top} & \tau^{3} e_{6}^{\top}
\end{array}\right), \quad \widetilde{G}_{3}^{\top}=\left(\begin{array}{lllll}
e_{1}^{\top} & e_{2}^{\top} & \tau e_{4}^{\top} & \tau^{2} e_{5}^{\top} & \tau^{3} e_{6}^{\top}
\end{array}\right),
\end{aligned}
$$

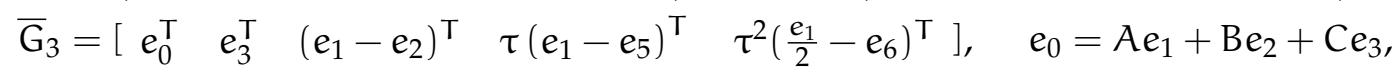

$$
\begin{aligned}
& \Omega_{3}=\left(e_{1}, e_{3}, e_{4}, e_{5}\right)^{\top}, \quad \widetilde{\Pi}_{1}=e_{1}-e_{3}, \quad \widetilde{\Pi}_{2}=e_{1}+2 e_{3}-6 e_{4}, \quad \widetilde{\Pi}_{3}=e_{1}-3 e_{3}+24 e_{4}-60 e_{5}, \\
& \widetilde{K}_{1}=\frac{\tau^{2}}{2} \widetilde{M}_{1} R_{1}^{-1} \widetilde{M}_{1}^{\top}+\frac{\tau^{2}}{4} \widetilde{M}_{2} R_{1}^{-1} \widetilde{M}_{2}^{\top}+\frac{\tau^{2}}{6} \widetilde{M}_{3} R_{1}^{-1} \widetilde{M}_{3}^{\top}+\tau \cdot \operatorname{sym}\left\{\widetilde{M}_{1} \widetilde{\Pi}_{1}+\widetilde{M}_{2} \widetilde{\Pi}_{2}+\widetilde{M}_{3} \widetilde{\Pi}_{3}\right\} \text {. }
\end{aligned}
$$

Proof. Construct a Lyapunov functional candidate as

$$
\begin{aligned}
V\left(x_{t}, t\right)= & G_{1}^{\top} X G_{1}+\int_{t-\tau}^{t} x^{\top}(s) Q_{1} x(s) d s+\int_{t-\tau}^{t}\left(\begin{array}{c}
x(s) \\
\dot{x}(s)
\end{array}\right)^{\top} \mathfrak{R}\left(\begin{array}{c}
x(s) \\
\dot{x}(s)
\end{array}\right) d s \\
& +\int_{t-\tau}^{t} \int_{\beta}^{t} x^{\top}(\alpha)\left(\tau W_{1}\right) x(\alpha) d \alpha d \beta+\int_{t-\tau}^{t} \int_{\theta}^{t} \int_{\lambda}^{t} \dot{x}^{\top}(s) R_{1} \dot{x}(s) d s d \lambda d \theta,
\end{aligned}
$$

where $X, Q_{1}, W_{1}, R_{1}, \Re$ are defined in Theorem 3.3. Following the similar line of the proof for Theorem 3.1, Theorem 3.3 is proved. It is omitted here. 
Table 1: The maximal allowable bounds of $h$ as $\tau$ is known.

\begin{tabular}{llll} 
References & $\tau=0.1$ & $\tau=0.5$ & $\tau=1.0$ \\
\hline$[9]$ & 1.7100 & 1.6718 & 1.6543 \\
{$[12]$} & 1.7844 & 1.7495 & 1.7201 \\
{$[15]$} & 1.8307 & 1.7755 & 1.7213 \\
{$[7]$} & 2.1229 & 2.1229 & 2.1229 \\
{$[11]$} & 2.2951 & 2.3471 & 2.3752 \\
{$[1](\mathrm{N}=1)$} & 1.8413 & 2.0954 & 2.2611 \\
{$[1](\mathrm{N}=3)$} & 2.1845 & 2.2973 & 2.3331 \\
{$[1](\mathrm{N}=5)$} & 2.2137 & 2.3210 & 2.3588 \\
Th 1 in[2] & 2.2021 & 2.2469 & 2.2670 \\
Th 2 in [2](N=1) & 1.8432 & 2.1078 & 2.2587 \\
Th 2 in [2](N=3) & 2.1886 & 2.3100 & 2.3452 \\
Th 2 in [2](N=5) & 2.2181 & 2.3331 & 2.3636 \\
[19] & 2.2959 & 2.3488 & 2.3769 \\
Analytical bounds & 2.2963 & 2.3491 & 2.3775 \\
\hline Theorem 3.1 & 2.2961 & 2.3491 & 2.3773 \\
\hline
\end{tabular}

Table 2: The maximal allowable bounds of the delay when $\tau=h$.

\begin{tabular}{lllll}
\hline$c$ & 0 & 0.1 & 0.3 & 0.5 \\
\hline$[4]$ & 4.47 & 3.49 & 2.06 & 1.14 \\
{$[5]$} & 4.35 & 4.33 & 4.10 & 3.62 \\
{$[18]$} & 4.47 & 4.35 & 4.13 & 3.67 \\
{$[8]$} & 4.47 & 4.42 & 4.17 & 3.69 \\
{$[14]$} & 4.63 & 4.57 & 4.29 & 3.75 \\
{$[17]$} & 5.30 & 5.21 & 4.85 & 4.20 \\
{$[3](\mathrm{N}=6)$} & 5.8613 & 5.7781 & 5.3946 & 4.6687 \\
{$[3](\mathrm{N}=7)$} & 5.8779 & 5.7910 & 5.4007 & 4.6703 \\
{$[3](\mathrm{N}=8)$} & 5.9287 & 5.8427 & 5.4417 & 4.6917 \\
{$[3](\mathrm{N}=9)$} & 6.1028 & 5.9816 & 5.5173 & 4.7247 \\
{$[10]$} & 6.117 & - & - & - \\
{$[19]$} & 6.1689 & 6.0339 & 5.5470 & 4.7374 \\
\hline Theorem 3.3 & 6.1707 & 6.0356 & 5.5479 & 4.7381 \\
\hline
\end{tabular}

\section{Numerical example}

In this section, we will demonstrate the superiority of our approach in the above section using the conventional neutral system by the following examples.

Example 4.1. Consider system (2.1) with the following parameters:

$$
A=\left(\begin{array}{cc}
-0.9 & 0.2 \\
0.1 & -0.9
\end{array}\right), \quad B=\left(\begin{array}{cc}
-1.1 & -0.2 \\
-0.1 & -1.1
\end{array}\right), \quad C=\left(\begin{array}{cc}
-0.2 & 0 \\
0.2 & -0.1
\end{array}\right)
$$

With different cases, we can obtain different maximum time delay $\mathrm{h}$ by using Matlab toolbox as listed in Table 1, compared with some existing references. From Table 1, we can see that the results in [2] and [1] are close to our results. However, the maximum of $h$ using Theorem 3.1 is nearly close to analytical bounds, which shows our approach is less conservative than the existing results. 
Example 4.2 ([4]). Consider system (3.1) with the state matrices listed as follows:

$$
A=\left(\begin{array}{cc}
-2 & 0 \\
0 & -0.9
\end{array}\right), \quad B=\left(\begin{array}{cc}
-1 & 0 \\
-1 & -1
\end{array}\right), \quad C=\left(\begin{array}{ll}
c & 0 \\
0 & c
\end{array}\right)
$$

with the positive scalar $c$ and $\tau=h$. With different $c$, we can obtain different maximum time delay $h$ by using Matlab toolbox as listed in Table 2, compared with the existing references. Clearly, the results of $h$ in $[4,7,8,18]$ and [14] are much more conservative than those in [3, 17] and Theorem 3.1. It is observed from Table 2 that our time-delay $h$ is much bigger than existing results with the less variables. This example shows again that our approach is effective and less conservative than the existing results.

Example 4.3. Consider the PEEC model introduced in [20] with $\tau=h$ and the parameter matrices listed as follows:

$$
A=100 \times\left(\begin{array}{ccc}
\theta & 1 & 2 \\
3 & -9 & 0 \\
1 & 2 & -6
\end{array}\right), \quad B=100 \times\left(\begin{array}{ccc}
1 & 0 & -3 \\
-0.5 & -0.5 & -1 \\
-0.5 & -1.5 & 0
\end{array}\right), \quad C=1 / 72 \times\left(\begin{array}{ccc}
-1 & 5 & 2 \\
4 & 0 & 3 \\
-2 & 4 & 1
\end{array}\right) .
$$

With our approach and using Matlab toolbox, the maximum time-delay $h$ compared to the existing results are listed in Table 3. It is quite evident that our result is the best.

\begin{tabular}{lccc}
\multicolumn{4}{l}{ Table 3: The maximal allowable delays $h$ for different values of $\theta$. } \\
\hline$\theta$ & -2.105 & -2.103 & -2.1 \\
\hline [6] & 1.0874 & 0.3709 & 0.2433 \\
[20] & 1.1413 & 0.3892 & 0.2533 \\
Th1 and Th2 in [16] & 1.3200 & 0.4917 & 0.3214 \\
Th3 and Th4 in [16] & 1.6978 & 0.5747 & 0.3749 \\
[19] & 1.7824 & 0.6030 & 0.3930 \\
\hline Theorem 3.3 & 1.7837 & 0.6034 & 0.3933 \\
\hline
\end{tabular}

\section{Conclusion}

In this paper, we have established two new inequalities which reduce the conservativeness of analysis for delay-dependent conditions. Based on the Lyapunov functional method and the two new inequalities, two sufficient conditions have been derived in terms of inequalities. Three numerical examples have been given to show the effectiveness and superiority of the proposed result.

\section{Acknowledgment}

This work is supported by National Nature Science Foundation of China under Grant 11461082, 11601474 and 11626093.

The author would like to thank the editors and the anonymous reviewers for their constructive comments and suggestions to improve the quality of the paper.

\section{References}

[1] Y.-G. Chen, S.-M. Fei, Z. Gu, Y.-M. Li, New mixed-delay-dependent robust stability conditions for uncertain linear neutral systems, IET Control Theory Appl., 8 (2014), 606-613. 1, 2.2, 1, 4.1

[2] L.-M. Ding, Y. He, M. Wu, C.-Y. Ning, Improved mixed-delay-dependent asymptotic stability criteria for neutral systems, IET Control Theory Appl., 9 (2015), 2180-2187. 1, 1, 4.1 
[3] M. Fang, J. H. Park, A multiple integral approach to stability of neutral time-delay systems, Appl. Math. Comput., 224 (2013), 714-718. 1, 2, 4.2

[4] E. Fridman, U. Shaked, Delay-dependent stability and $\mathrm{H}_{\infty}$ control: constant and time-varying delays, Internat. J. Control, 76 (2003), 48-60. 1, 2, 4.2

[5] Q.-L. Han, Robust stability of uncertain delay-differential systems of neutral type, Automatica J. IFAC, 38 (2002), 719723. 2

[6] Q.-L. Han, On robust stability of neutral systems with time-varying discrete delay and norm-bounded uncertainty, Automatica J. IFAC, 40 (2004), 1087-1092. 3

[7] Q.-L. Han, On stability of linear neutral systems with mixed time delays: a discretized Lyapunov functional approach, Automatica J. IFAC, 41 (2005), 1209-1218. 1, 2.4, 3, 3, 1, 4.2

[8] Y. He, Q.-G. Wang, C. Lin, M. Wu, Augmented Lyapunov functional and delay-dependent stability criteria for neutral systems, Internat. J. Robust Nonlinear Control, 15 (2005), 923-933. 1, 2, 4.2

[9] Y. He, M. Wu, J.-H. She, G.-P. Liu, Delay-dependent robust stability criteria for uncertain neutral systems with mixed delays, Systems Control Lett., 51 (2004), 57-65. 1, 1

[10] C.-Y. Kao, A. Rantzer, Stability analysis of systems with uncertain time-varying delays, Automatica J. IFAC, 43 (2007), 959-970. 2

[11] X.-G. Li, X.-J. Zhu, A. Cela, A. Reama, Stability analysis of neutral systems with mixed delays, Automatica J. IFAC, 44 (2008), 2968-2972. 1, 1

[12] X.-G. Liu, M. Wu, R. Martin, M.-L. Tang, Stability analysis for neutral systems with mixed delays, J. Comput. Appl. Math., 202 (2007), 478-497. 1, 1

[13] P. G. Park, W. I. Lee, S Y. Lee, Auxiliary function-based integral inequalities for quadratic functions and their applications to time-delay systems, J. Franklin Inst., 352 (2015), 1378-1396. 1

[14] M. N. A. Parlakçı, Robust stability of uncertain neutral systems: a novel augmented Lyapunov functional approach, IET Control Theory Appl., 1 (2007), 802-809. 2, 4.2

[15] W. Qian, J. Liu, Y.-X. Sun, S.-M. Fei, A less conservative robust stability criteria for uncertain neutral systems with mixed delays, Math. Comput. Simulation, 80 (2010), 1007-1017. 1, 1

[16] J. Sun, G. P. Liu, On improved delay-dependent stability criteria for neutral time-delay systems, Eur. J. Control, 15 (2009), 613-623. 3

[17] J. Sun, G. P. Liu, J. Chen, Delay-dependent stability and stabilization of neutral time-delay systems, Internat. J. Robust Nonlinear Control, 19 (2009), 1364-1375. 1, 2, 4.2

[18] M. Wu, Y. He, J.-H. She, New delay-dependent stability criteria and stabilizing method for neutral systems, IEEE Trans. Automat. Control, 49 (2004), 2266-2271. 1, 2, 4.2

[19] L.-L. Xiong, H.-Y. Zhang, Y.-K. Li, Z.-X. Liu, Improved stabilization criteria for neutral time-delay systems, Math. Probl. Eng., 2016 (2016), 13 pages. 1, 2, 3

[20] D. Yue, Q.-L. Han, A delay-dependent stability criterion of neutral systems and its application to a partial element equivalent circuit model, IEEE Trans. Circuits Syst. II, Exp. Briefs, 51 (2004), 685-689. 4.3, 3

[21] H.-B. Zeng, Y. He, M. Wu, J.-H. She, Free-matrix-based integral inequality for stability analysis of systems with timevarying delay, IEEE Trans. Automat. Control, 60 (2015), 2768-2772. 1

[22] H.-B. Zeng, Y. He, M. Wu, J.-H. She, New results on stability analysis for systems with discrete distributed delay, Automatica J. IFAC, 60 (2015), 189-192. 1, 2.4

[23] N. Zhao, C. Lin, B. Chen, Q.-G. Wang, A new double integral inequality and application to stability test for time-delay systems, Appl. Math. Lett. 65 (2017), 26-31. 2.4 